\title{
Efectividad de enjuagues bucales en el tratamiento dental durante la pandemia COVID-19
}

\author{
Effectiveness of mouthwashes in dental treatment during the COVID-19 \\ pandemic
}

\author{
Karla Ivohnne Pedraza Maquera ${ }^{\text {a }}$, Caroll Johana Uberlinda Lévano Villanueva ${ }^{2 b}$ \\ iD https://orcid.org/0000-0002-8156-833X \\ iD https://orcid.org/0000-0003-1072-374 \\ ${ }^{1}$ Escuela Profesional de Odontología, Universidad Nacional Jorge Basadre Grohmann. Tacna, Perú. \\ ${ }^{2}$ Investigador independiente. Tacna, Perú. \\ ${ }^{a}$ Docente, Maestro en Odontología con mención en Patología. \\ ${ }^{\mathrm{b}}$ Maestro en Ciencias con Mención en Salud Pública.
}

\begin{abstract}
Resumen
La pandemia por coronavirus 2019 (COVID-19) originada en Wuhan, China, se ha extendido a todo el mundo, ocasionando una crisis para la salud pública a nivel mundial. Las medidas de control de infecciones son necesarias para evitar que el virus se propague aún más, y para ayudar a controlar la situación epidémica. Debido a las características de los entornos dentales, el riesgo de infección cruzada puede ser alto, tanto en pacientes como en odontólogos. Dada la necesidad de una búsqueda de medios de control para las infecciones por COVID-19, se realizó una revisión bibliográfica de artículos publicados relacionados con la efectividad de los enjuagues bucales, como profilácticos en la actividad virucida, para reducir la cantidad de microorganismos diseminados por medio de aerosol generado a través de procedimientos dentales; a su vez disminuir la transmisión de la infección. El propósito de esta revisión fue recopilar la información existente en los últimos años sobre los criterios para el uso de enjuagues bucales en la práctica dental, que puede coadyuvar en la necesidad de prevención de los odontólogos a nivel mundial.
\end{abstract}

Palabras clave: Enjuague bucal, COVID-19, transmisión de infección, SARS-CoV-2 y coronavirus.

\begin{abstract}
The 2019 coronavirus disease pandemic (COVID-19), which originated in Wuhan, China, has spread exponentially to all parts of the world and has become a global public health crisis. Infection control measures are necessary to prevent the virus from spreading further, and to help control the epidemic situation. Due to the characteristics of dental settings, the risk of cross infection may be high both in patients and dentists. Being a necessity the search of control means for the infections by COVID-19. A bibliographical review of articles published was carried out, related to the effectiveness of the mouthwashes as prophylactic in the virucidal activity, to reduce the amount of microorganisms spread by means of aerosol generated through dental procedures, in turn to diminish the transmission of the infection. The purpose of this review was to collect the existing information in recent years on criteria for the use of mouthwashes in dental practice, which may contribute to the need for prevention by dentists worldwide.
\end{abstract}

Keywords: Mouthwash, COVID-19, transmission of infection, SARS-CoV-2 y coronavirus. 


\section{Introducción}

El nuevo coronavirus se identificó en Wuhan, China, en diciembre de 2019, en pacientes con neumonía de origen desconocido. El 9 de enero de 2020, la Organización Mundial de la Salud (OMS) declaró el descubrimiento de un nuevo coronavirus, llamándole finalmente COVID-19. Actualmente, expandido por todo el mundo, el COVID-19 tiene 5 millones de infectados y 332.000 fallecidos; cifras que van en aumento, y son alarmantes para la salud pública mundial; y una alerta para el ámbito de la odontología, un grupo profesional con una alta probabilidad de contagio por la carga viral SARS CoV-2, o COVID-19, en la saliva de los pacientes infectados; por este motivo es que existe la necesidad de aumentar las investigaciones sobre la presencia de COVID-19 en fluidos orales, y su impacto en la transmisión de este virus, que es crucial para mejorar las estrategias efectivas de prevención en los procedimientos de generación de aerosoles. La saliva juega un papel fundamental en la transmisión del COVID-19; por lo que los enjuagues bucales podrían ser parte crucial para mejorar las estrategias efectivas de prevención; esa posibilidad motivó la realización de este artículo de investigación, con el objetivo de encontrar un esclarecimiento acerca del uso de los enjuagues bucales en infecciones respiratorias similares al SARS CoV, y ahora el SARS-CoV-2; justificándose en la preocupación de buscar información sobre estrategias de prevención en la atención dental para evitar el contagio paciente-odontólogo.

Se realizó una busqueda de artículos científicos vía internet, en buscadores como Google Scholar, Science Direct, Elsevier, PudMed. Los criterios utilizados para la selección de artículos de relevacia estuvieron en concordancia con las palabras clave: mouthwashes, mouth rinse, enjuagatorios bucales, COVID-19, coronavirus, SARS-Cov-2, SARS CoV; estos no se discriminaron por idioma, país o tipo de estudio. Para la selección se revisaron los artículos completos a fin de verificar que el contenido tuviera relación con el objetivo del trabajo, considerando que la informacion acerca del coronavirus (COVID-19) es limitada se consideraron artículos publicados desde el año 2003 al 2020. La relevancia de los artículos más antiguos, como los de Li W. (2003), Kuba K. (2005), Kariwa (2006), está en los resultados en base a cepas como el SARS y el MERS que tienen relacion con el SARS-CoV y el SARS-CoV-2. Se seleccionaron 25 artículos que fueron los que tuvieron relación entre sí y con el tema que aborda este artículo.

\section{La infección del COVID-19}

La infección de la célula hospedadora por el virus es importante para el desarrollo y evolución del COVID-
19; la enzima convertidora de angiotensina 2 (ACE-2) es la principal proteína de membrana que interactúa con el virus SARS-CoV-2 para infectar a las células, estos receptores están en el pulmón, intestino, riñón y lengua (1-3).

El COVID-19 se detecta en la cavidad oral, debido a la saliva de las glándulas salivales mayores y menores infectadas, lo que sugiere que las células de las glándulas salivales podrían ser una fuente fundamental de este virus en la saliva (4).

Es importante tener en cuenta que Kutter et al. (5) (2018) concluyeron que los aerosoles con agentes patógenos, como el SARS-COV, pueden viajar $1.8 \mathrm{~m}, \mathrm{y}$ que tienen capacidad de sobrevivir en diferentes superficies; asimismo, al hacer el isopado de las superficies de las habitaciones con pacientes que tenían SARS dieron positivo al PCR. La distancia de diseminación y la viabilidad del virus (que es hasta 3 horas), además de su estabilidad en superficies de acero y plástico (que es alrededor de 72 horas a más) juegan un papel importante en la trasmisión de la infección. El enjuague bucal es un medio importante de reducción de la carga viral de la saliva, lo que reduce el contagio directo y cruzado del COVID-19(6).

\section{Efectividad de los enjuagues bucales}

El enjuague bucal es una solución que se usa para mantener la higiene bucal y para eliminar las bacterias. Los enjuagatorios bucales son un procedimiento necesario y efectivo para reducir la carga de microorganismos en aerosoles orales. Marui et al. (2019), citado por Ge et al. en el 2020, mostraron que el uso de la clorhexidina (CHX), aceites esenciales y cetilpiridinio cloruro (CPC) redujo en un $68.4 \%$ de unidades formadoras de colonias en aerosol dental, pero no hay evidencia clínica que alguna de ellas sea eficaz en COVID-19 (7).

La Comisión Nacional de Salud del gobierno chino elaboró la guía para el diagnóstico y tratamiento de la neumonía por coronavirus, e indicó que la clorhexidina al $0.12 \%$ que se usa comúnmente como enjuague bucal, en la práctica, no es efectiva para el SARS-CoV-2; este virus es susceptible a la oxidación, por lo que los enjuagues bucales sugeridos son el peróxido de hidrógeno al $1 \%$ o yodopovidona al $0.2 \%(7-9,22-24)$.

La povidona yodada (PVP-I) es conocida como microbicida de amplio espectro contra bacterias, hongos, protozoos y virus; es, además, un complejo soluble en agua. El yodo elemental está unido al vehículo polivinilpirrolidona, y para obtener el agente antimicrobiano, o el yodo activo, necesita de un medio acuoso en equilibrio químico; mientras que el complejo de yodo enlazado forma reservorios para su liberación; de esta forma se mantiene la actividad microbicida, y los efectos citotóxicos de altas concentraciones de yodo se reducen $(9,10)$. La potencia oxidativa de PVP-I permite que el yodo liberado reaccione rápidamente con grupos 
funcionales de aminoácidos y nucleótidos, así como con dobles enlaces de ácidos grasos; lo que resulta en una variada destrucción de varias estructuras y enzimas de microbios y virus (10).

La aplicación de aerosol liposomal de PVP-I en la mucosa nasal, no disminuye la función nasal ni presenta daño en el epitelio ciliado de múltiples capas de la nariz. La mejora de varios parámetros de la función nasal bajo PVP-I liposomal sugiere un aclaramiento mucociliar mejorado y actividad mucolítica de yoduro debido a la aplicación local de los constituyentes (11).

La PVP-I tiene una mayor actividad virucida que otros agentes antisépticos de uso común, como la clorhexidina y el cloruro de benzalconio. Se ha demostrado que es activo in vitro contra los coronavirus que han causado epidemias en las últimas dos décadas, como el SARS-CoV, que causó la epidemia del síndrome respiratorio agudo severo (SARS) de 2002-2003 y el MERS-CoV, el agente responsable de causar la epidemia del síndrome respiratorio del Medio
Oriente (MERS) de 2012-2013 (11,12).

El SARS-CoV-2 es altamente homólogo con el SARS$\mathrm{CoV}$, el cual se le considera un pariente cercano (12). Eggers et al. (2015) $(13,14)$ mostraron que la concentración efectiva más baja de PVP-I para el MERS-CoV fue del $1 \%$, cuando se usó durante 30 segundos en condiciones "sucias", con una reducción de la actividad viral de $\geq 99.99 \%$; sin embargo, esto no fue efectivo al $0.1 \%$, posteriormente, la concentración más baja probada, y aún efectiva contra los coronavirus fue de $0.23 \%$.

Kariwa et al. (2006) (15) demostraron en un estudio in vitro, que el PVP-I, en sus diferentes presentaciones, es efectivo a diferentes concentraciones ante el SARS$\mathrm{CoV}$, después de los 2 minutos, como se observa en la tabla 1 el Isodine gárgaras no fue muy efectivo al minuto, sino a los 2 minutos, en comparación con el Isodine Nodo Fresh, que, pese a su concentración más baja, tuvo efectividad al minuto de aplicado.

Tabla 1. Eficacia de los productos PVP-I para SARS-CoV.

\begin{tabular}{|c|c|c|c|}
\hline \multirow{2}{*}{ Reagent or treatment } & \multirow{2}{*}{$\begin{array}{c}\text { Final PVP-I } \\
\text { concentration, \% }\end{array}$} & \multicolumn{2}{|c|}{ Virus titer after treatment, TCID $_{50} / \mathrm{ML}$} \\
\hline & & $60 \mathrm{~S}$ & $120 \mathrm{~s}$ \\
\hline Control & 0 & $1.17 \times 10^{6}$ & n.d \\
\hline Isodine & 1 & 95.1 & u.d \\
\hline Isodine Gargle & 0.47 & 190 & u.d \\
\hline Isodine Scrub & 1 & u.d & n.d \\
\hline Isodine Palm & 0.25 & u.d & n.d \\
\hline Isodine Nodo Fresh & 0.23 & u.d & n.d \\
\hline Ethanol (final 35\%) & - & u.d & n.d \\
\hline
\end{tabular}

Fuente: Kariwa H. $(15,18)$.

El tratamiento de etanol al $70 \%$ durante 1 minuto, también redujo la infectividad del virus por debajo del nivel detectable. Estos resultados fuertemente indican que los productos PVP-I, y el 70\% de etanol son efectivos para la inactivación del SARS-CoV $(15,8)$.

En otras investigaciones de revisión Baker et al. (2020) (16) sugieren la reutilización de compuestos de amonio cuaternario en posibles tratamientos para COVID-19, como el cloruro de amonio, cloruro de cetilpiridinio y miramistin. Estos compuestos tienen un agente lisosomotrópico, que puede producir el deseado efecto antiviral, pero aún no se han probado contra el SARS$\mathrm{CoV}-2$ in vitro o en la clínica.

El cloruro de cetilpiridinio es una molécula simple, barata, segura, clínicamente aprobada, ampliamente accesible, que podría ingresar a ensayos inmediatamente. Se encuentra en forma de enjuague oral y aerosol nasal, y pueden ayudar a combatir el virus en su punto de entrada y reducir la transmisión del SARS-CoV-2, pudiéndose agregar a los champús y limpiadores corporales en bajas concentraciones. La miramistina, que pertenece al grupo de antisépticos catiónicos, es un medicamento también utilizado en odontología, con amplia gama de actividades biológicas, como antiviral contra el VIH, influenza, herpes y el SARS. El cloruro de amonio tiene un mecanismo de acción que eleva el $\mathrm{pH}$ endocítico y lisosómico que comparte con la cloroquina; es eficaz en murine, coronavirus, hepatitis C (16).

Carrouel et al. (2020) (17) recomiendan examinar el efecto de enjuagues bucales con B-ciclodextrina combinada con citrox en la prevención de infecciones y progresión del COVID-19; en el caso del citrox para el tratamiento específico de COVID-19, los flavonoides como inhibidores de la proteasa, similares a la quimotripsina coronavirales, tienen una función esencial para replicación coronavírica; además, como el SARS-CoV-2 es vulnerable a la oxidación, se recomienda usar un enjuague bucal que contenga agentes oxidantes como citrox para reducir la carga 
viral salival de la microbiota oral, incluido el posible transporte del SARS-CoV-2.

Carrouel et al. sugieren enjuagues bucales con Bciclodextrina anfifílica para el tratamiento específico de COVID-19, que contienen agentes oxidativos; para reducir la carga microbiana, dado que contienen moléculas de azúcar modificadas que atraen los virus antes de inactivarlos irreversiblemente, irrumpiendo en la capa externa de un virus al simple contacto. Esta propiedad puede potencialmente explotarse para la reducción de la carga viral en la cavidad oral.

Enjuagues bucales con ciclodextrinas combinadas con citrox para el tratamiento específico de COVID-19 en uso de enjuagues bucales y/o aplicaciones nasales, podrían proporcionar un valioso tratamiento complementario para reducir la carga viral del SARS$\mathrm{CoV}-2$ y reducir la microbiota nasofaríngea al momento de estornudar, o en la emisión de aerosoles al tratamiento dental; dichos productos han sido testeados, pero es necesario iniciar ensayos clínicos para evaluar sus efectos sobre el SARS-CoV-2 $(9,17)$.

Manger et al. indican que la eliminación mecánica de la placa con el cepillado dental, como una intervención de higiene oral en pacientes hospitalarios, reduce las complicaciones de la neumonía. La intervención química con clorhexidina o PVP-I reduce el riesgo de neumonía asociada al ventilador (VAP) en cuidados intensivos (19). El cuidado de la salud oral se está tratando como un "Servicio de Cenicienta" en la comunidad y entre los pacientes del hospital. El régimen de higiene oral profiláctico oportuno, y la conciencia de los resultados nocivos de la mala salud oral pueden mejorar en gran medida la salud de los pacientes vulnerables, y cerrar la brecha entre la salud oral y general para una mejor atención al paciente $(20$, $21,23,25)$.

\section{Discusión}

La literatura disponible enumera sustancias activas antivirales, pero no se puede concluir que todas ellas son efectivas para el virus SARS-CoV-2, debido a que ninguna de ellas en la actualidad tiene evidencia cientifica de ser efectiva in vivo y mucho menos como enjuague bucal. Lo que se describe es una serie de datos obtenidos en el pasado con virus similares en estudios in vitro sobre muestras recogidas de superficies inanimadas en el campo de la desinfección. Es importante reconocer que los autores como Kariwa (2006), Egeers (2015), Kirk-Bayley (2020),
Pattanshetty (2020) coinciden en que la yodopovidona al $0.2 \%$ y el peroxido de hidrógeno al $1 \%$ son efectivos para el SARS-CoV in vitro, pero que es necesario probarla en la clínica y determinar sus efectos sobre el COVID-19, además de establecer una concentración letal para el virus, pero no dañina para el paciente. Otras de las soluciones como enjuague bucal preoperatorio para reducir la carga viral recomendadas por Baker et al. (2020), Ge Z (2020) y Basso (2020), quienes citan a Li Zhiyong, es el cloruro de cetilpiridinio en una concentración entre el $0.05 \%$ y el $0.10 \%$ que ha demostrado ser eficaz contra el virus MERS-CoV; un coronavirus responsable del síndrome respiratorio del Medio Oriente. Pero que pese a ello no es posible determinar su eficacia en el virus causante de la pandemia actual. Por otro lado Carrouel et al. (2020) recomiendan examinar el efecto de enjuagues bucales con B-ciclodextrina combinada con citrox, pues ambas sustancias tienen efectos oxidativos sobre los virus, pero aún no han sido testeados en pacientes con COVID-19. Es necesario que se hagan pruebas en pacientes COVID-19, de tal manera que podamos hacer uso de estas sustancias, o de otras no expuestas en este artículo, para disminuir la carga viral en la saliva, y así implementar protocolos a la luz de las evidencias clínicas.

\section{Conclusión}

La investigación da a conocer los diferentes estudios que nos dan una visión más amplia de nuestra inquietud, cumpliendo el objetivo de contribuir en la indagación de estrategias de prevención de infección por el COVID19.

Según las revisiones bibliográficas del artículo, existen sustancias que pueden ser usadas como enjuagues bucales; tal es el caso de la yodopavidona, que es una sustancia que ha demostrado tener propiedades virucidas in vitro contra el SARS-CoV; así mismo, hay varios autores que proponen, en medio de esta pandemia, otras opciones fundamentadas como los amonios cuaternarios, el citrox en combinacion con la B- ciclodextrina anfifílica; lo que nos brinda otras opciones diferentes a la yodopovidona y el peróxido de hidrógeno, que son muy recomendados para reducir la carga microbiana-viral de la saliva. No obstante, es necesario que se realicen investigaciones con ensayos clínicos en pacientes infectados con COVID-19 para corroborar la efectividad que tienen estos compuestos en boca y orofaringe reduciendo la carga viral en la saliva. 


\section{Referencias bibliográficas}

1. Pérez-Domínguez, M., Pérez-Ybarra L. SARSCoV-2 en saliva: potencial vía de contagio e implicaciones en el tratamiento del paciente odontológico. Odous Científica. 2020; 21(1): 77-88 77 Online ISSN: 2665-0193 - Print ISSN: 1315-2823.

2. Li W, Moore MJ, Vasilieva N, Sui J, Wong SK, Berne MA et al. Angiotensin-converting enzyme 2 is a functional receptor for the SARS coronavirus. Nature. 2003; 426(6965):450-4. [On line] [Accessed April 27 2020]. Available from: https://doi.org/10.1038/nature02145

3. Kuba K, Imai Y, Rao S, Gao H, Guo F, Guan B. A crucial role of angiotensin converting enzyme 2 (ACE2) in SARS coronavirus- induced lung injury. Nat Med. 2005 Aug; 11(8):875-9. [On line] [Accessed April 27 2020]. Available from: https://doi.org/10.1038/nm1267

4. Sabino-Silva, R., Jardim, A.C.G. \& Siqueira, W.L. Coronavirus COVID-19 impacts to dentistry and potential salivary diagnosis. Clin Oral Invest 24, 1619-1621 (2020). [On line] [Accessed April 27 2020]. Available from: https://doi.org/10.1007/s00784-020-03248-x

5. Kutter JS, Spronken M, Fraaij P, Fouchier R. Herfst S. Transmission routes of respiratory viruses amonghumans. Current Opinion in Virology. 2018; 28: p. 142-151. [On line] [Accessed April 27 2020]. Available from: https://www. ncbi.nlm.nih.gov/pubmed/29452994

6. Van Doremalen N, Bushmaker T, Morris DH, Holbrook MG, Gamble A, Williamson B $++\mathrm{N}$ et al. Aerosol and Surface Stability of SARS-CoV2 as Compared with SARS-CoV-1. The New England journal of medicine. 2020 Mar 17. [On line] [Accessed April 27 2020]. Available from: https://doi.org/10.1056/NEJMc2004973

7. Ge, Z., Yang, L., Xia, J. et al. Possible aerosol transmission of COVID-19 and special precautions in dentistry. J. Zhejiang Univ. Sci. B (2020). [On line] [Accessed April 27 2020]. A v a i 1 a b 1 e F r o m : https://doi.org/10.1631/jzus.B20100100

8. Suárez S, Campuzano R, Dona Vidale M, Garrido E, Giménez T. Recomendaciones para prevención y control de infecciones por SARSCoV-2 en odontología. RO [Internet]. 8 de abril de 2020 [Citado el 27 de abril 2020]; 22(2):5-32. D i s p o n i b l e e $n$ : http://revistadigital.uce.edu.ec/index.php/odont ologia/article/view/2211

9. BASSO, M, Bordini, G., Bianchi, F., Prosper, L., Testori, T., \& Del Fabbro, M. Utilizzo di collutori preoperativi contro il virus SARSCoV-2 (COVID-19): revisione della letteratura e raccomandazioni cliniche. Rivisti Online Quintessencce Publishing-Italia 2020, vol. 1, p. $20 \quad$ D i s p o i bili i n : https://www.quintessenzaedizioni.com/utilizzo -collutori-preoperativi-contro-virus-sars-covcovid-revisione-della-letteraturaraccomandazioni-cliniche/d/30.html

10. Eggers, Cols. Povidone-iodine hand wash and hand rub products demonstrated excellent in vitro virucidal efficacy against Ebola virus and modified vaccinia virus Ankara, the new European test virus for enveloped viruses. BMC Infectious Diseases. 2015; 15. [On line] [Accessed April 27 $\left.\begin{array}{llll}2 & 0 & 2 & 0\end{array}\right]$. A va i 1 a b 1 e from: ht t p s://ww w. nc bi.n $1 \mathrm{~m} . \mathrm{nih}$. Gov/PubMed/26381737 or DOI: 10.1186/s12879-015-1111-9.

11. GLUCK, U., et al. A clinical study on the tolerability of a liposomal povidone-iodine nasal spray: implications for further development. ORL, 2007, vol. 69, no 2, p. 92-99. [On line] [Accessed April 27 2020]. Available $\mathrm{f} \quad \mathrm{r} \quad \mathrm{o} \quad \mathrm{m}$ : https://www.karger.com/Article/Abstract/9775 8\# or https://doi.org/10.1159/000097758

12. Kirk-Bayley, Justin and Combes, James and Sunkaraneni, Vishnu and Challacombe, Stephen, The Use of Povidone Iodine Nasal Spray and Mouthwash During the Current COVID-19 Pandemic May Reduce Cross Infection and Protect Healthcare Workers (March 28, 2020). [On line] [Accessed April 27 $20020]$. Ava i 1 a b 1 e a t S S R N : https://ssrn.com/abstract=356309213

13. Eggers M, Koburger-Janssen T, Eickmann M, Zorn J. Rapid and effective virucidal activity of povidone-iodine products against Middle East respiratory syndrome coronavirus (MERSCoV) and modified vaccinia virus ankara (MVA). Infect Dis Ther. 2015 Dec;4(4):491-501 [On line] [Accessed April 27 2020]. Available from: https://www.ncbi.nlm.nih.gov/pubmed/264162 14

14. Eggers M, Koburger-Janssen T, Eickmann M, et al. In Vitro Bactericidal and Virucidal E ffic a c y of Povidone-Iodine 
Gargle/Mouthwash against Respiratory and Oral Tract Pathogens. Infectious diseases and therapy 2018; 7:249-59. [On line] [Accessed April 27 2020] Available from: https://www.ncbi.nlm.nih.gov/pubmed/29633 177

15. Kariwa H, Fujii N, Takashima I. Inactivation of SARS coronavirus by means of povidoneiodine, physical conditions and chemical reagents. Dermatology. 2006;212(Supp1 1):119-123. [On line] [Accessed May 32020 ]. A va i 1 a b 1 e fro m : https://www.ncbi.nlm.nih.gov/pubmed/16490 989

16. Baker, N., Williams, A. J., Tropsha, A., \& Ekins, S. Repurposing Quaternary Ammonium Compounds as Potential Treatments for COVID-19. (2020, April 14). [On line] [Accessed May 3 2020]. Available from: https://doi.org/10.31219/osf.io/ehsn3

17. Carrouel F, Conte MP, Fisher J, Gonçalves | LS, and Dussart C, and Llodra JC, et al. COVID-19: A Recommendation to Examine the Effect of Mouthrinses with $\beta$-Cyclodextrin Combined with Citrox in Preventing Infection and Progression. Journal of Clinical Medicine. MDPIAG; 2020 April 15; 9(4):1126. [On line] [Accessed May 4 2020] Available from: file://C:/Users/Villa/Downloads/jcm-0901126-v2.pdf

18. García- Gargallo M., Zurlohe M, Montero e, Alonso B, Serrano J, Sanz M y Cols. Evaluación de nuevas formulaciones de enjuague bucal a base de clorhexidina y cloruro de cetilpiridinio adyuvantes de la escala y el alisado radicular: estudio piloto. 2017 noviembre; 15 (4): 269-279. [On line] [Citado el 4 de mayo 2020]. Disponible en: https://www.ncbi.nlm.nih.gov/pubmed/27762 095

19. Manger, D., Walshaw, M., Fitzgerald, R. et al. Evidence summary: the relationship between oral health and pulmonary disease. $\mathrm{Br}$ Dent $J$ 222, 527-533 (2017). [On line] [Accessed May 4 2020] Available from: https://doi.org/10.1038/sj.bdj.2017.31

20. Pattanshetty S, Narayana A, Radhakrishnan R. Povidoneionide gargle as a prophylactic intervention to interrupt the transmission or Sars-Cov2- [On line] [Accessed May 4 2020]. A v a i l a b l e From : https://onlinelibrary.wiley.com/doi/epdf/10.1 111/odi.13378

21. Lo Giudice R. The Severe Acute Respiratory Syndrome Coronavirus-2 (SARS CoV-2) in Dentistry. Management of Biological Risk in Dental Practice. International Journal of Environmental Research and Public Health [Internet]. MDPI AG; 2020 Apr 28; 17(9):3067. [Accessed May 4 2020]. Available $\mathrm{f}$ r o m : http://dx.doi.org/10.3390/ijerph17093067

22. Chinese Clinical Guidance for COVID-19 Pneumonia Diagnosis and Treatment, 7th ed.; China National Health Commission: Beijing, China, 2020; [accessed May 4 2020]. Ava i a ble on line: http://kjfy.meetingchina.org/msite/news/sho $\mathrm{w} / \mathrm{cn} / 3337 . \mathrm{html}$

23. ARAYA, S. C. Consideraciones para la Atención de Urgencia Odontológica y Medidas Preventivas para COVID-19 (SARSCoV 2). Int. J. Odontostomat, 2020, 14 (3): 268-270. 2020 [Interne] [Citado el 4 de mayo d e $1 \quad 2 \quad 0620]$. D i s p o n i b l e en:http://www.ijodontostomatology.com/es/a rticulo/consideraciones-para-la-atencion-deurgencia-odontologica-y-medidaspreventivas-para-covid-19-sars-cov-2

24. Sigua-Rodríguez, E. A., Bernal-Pérez, J. L., Lanata-Flores, A. G., Sánchez-Romero, C., Rodríguez-Chessa, J., Haidar, Z., \& OLATE, S. COVID-19 y la Odontología: una Revisión de las Recomendaciones y Perspectivas para Latinoamérica. Int. J. Odontostomat, 2020, 14(3), 299-309. [Internet] [Citado el 4 de mayo $\left.\begin{array}{llll}2 & 0 & 2 & 0\end{array}\right]$. D i s p o n i b l e e n : http://www.ijodontostomatology.com/wpcontent/uploads/2020/04/2020_v14n3_007.p df

25. MENG, L.; HUA, F.; BIAN, Z. Coronavirus disease 2019 (COVID-19): emerging and future challenges for dental and oral medicine. Journal of Dental Research, 2020, vol. 99, no 5, p. 481-487. [On line] [Accessed May 4 $\left.\begin{array}{llll}2 & 0 & 2 & 0\end{array}\right] \quad A$ va i 1 a b 1 e from : https://journals.sagepub.com/doi/full/10.1177 /0022034520914246
Correspondencia:

karlaivho@hotmail.com
Fecha de recepción : 18 de marzo de 2020

Fecha de aceptación : 28 de mayo de 2020 